\title{
Wear Law and Parameter Optimization Study on the Split Cone of a Vertical Shaft Impact Crusher
}

\author{
Jie Yang, Feifei Feng $\mathbb{D}$, Junxu Ma, and Jinfa Shi $\mathbb{D}$ \\ North China University of Water Resources and Electric Power, Zhengzhou 450045, China \\ Correspondence should be addressed to Feifei Feng; 1573865218@qq.com and Jinfa Shi; shijinfa@ncwu.edu.cn
}

Received 31 March 2021; Revised 21 June 2021; Accepted 3 July 2021; Published 13 July 2021

Academic Editor: Pietro Bia

Copyright (c) 2021 Jie Yang et al. This is an open access article distributed under the Creative Commons Attribution License, which permits unrestricted use, distribution, and reproduction in any medium, provided the original work is properly cited.

\begin{abstract}
During the sand-making process, the wear of the split cone will affect the dynamic balance of the rotor and the sand-making effect of the crusher. This paper focuses on experiments and simulations with the rotor of a vertical shaft impact crusher. A wear model for predicting the wear value of the split cone is established and its effectiveness is verified. The orthogonal test of $L_{16}\left(4^{4}\right)$ is designed. By using the range analysis method, the influence of the rotor speed, the feed height, the installation angle, and the number of the guide plate on the wear resistance of the split cone are studied. By using the multiple linear regression analysis method, the optimal parameter combination of each factor is obtained. The results of this paper show that the wear resistance of the split cone is the best when the installation angle of the guide plate is $40^{\circ}$, the installation number of the guide plate is 3 , the rotor speed is $2800 \mathrm{r} / \mathrm{min}$, and the feed height is $40 \mathrm{~mm}$.
\end{abstract}

\section{Introduction}

The rotor of the vertical shaft impact crusher will be worn to varying degrees under the impact of external particles. When the particles enter the rotor from the feed port, the split cone, as the main component inside the rotor, bears the impact of the particles and will inevitably wear out. It belongs to one of the vulnerable parts. If it is not replaced in time, it will affect the diversion effect of the particle and makes the particles distribution uneven between the guide plates, which will affect the dynamic balance of the rotor and the sand-making effect of the crusher $[1,2]$.

Relevant researchers have carried out related work through experimental and simulation research methods and achieved good results. In terms of wear calculation model, Gui [3] explained the importance of wear design calculation and comprehensively and systematically introduced the wear model proposed by Professor J.F. Archard, a wellknown expert in friction. Grewal et al. [4] proposed a mathematical model for predicting the erosion of brittle materials and coating slurries. An erosion model was established based on the theory of Hertz contact stress and fracture mechanics, which accurately predicted the erosion rate of the coating. Finnie $[5,6]$ proposed a Finnie wear model for the erosion of plastic materials. This model can quantitatively predict the erosion of plastic metal when the particles impact the surface of the plastic metal surface at a certain impact angle. Guan and others [7] studied the impact of fretting wear of thin-walled tube supports in clamps in nuclear power plants. In terms of improving wear resistance, a researcher [8] introduced a company that used wear-resistant austenitic manganese steel as the wear-resistant material of the crusher to improve the wear resistance of wear parts and increase their service life. Yin and others [9] improved the wear resistance of the accelerator plate by changing the structural and production parameters of the rotor. In terms of wear simulation, Liu and Yang [10] used simulation methods to effectively predict the amount of wear on the disc hob. Xu and others [11] used DEM to simulate the movement of materials and effectively predicted the wear of the SAG lining surface. Zhang and others [12] used the discrete element method and regression analysis method to optimize the structural parameters of the rotor to improve the acceleration effect of the rotor on the particles. $\mathrm{Lu}$ and Houzhiqiang [13] used the discrete element method to analyze the wear forms and wear characteristics of the wear- 
resistant parts of the rotor under different working conditions.

In addition to the above-mentioned scholars, other domestic and foreign scholars have also studied the vertical shaft impact crusher [14-16]. However, the influence of the structural parameters and production parameters of the rotor on the wear of the split cone has not been explored. In this paper, the wear model of the split cone is established to estimate the wear value of wear of the split cone. The simulation test is arranged by orthogonal test and the discrete element software EDEM is used for simulation to obtain the simulation results. To explore the influence of the structural parameters and production parameters of the rotor on the wear of the split cone by using the method of range analysis, the optimal parameter combination of various factors is found to reduce the wear of the split cone by using the method of regression analysis. It provides some theoretical guidance for improving the wear-resistant performance of the split cone.

\section{The Establishment of Wear Model of Split Cone}

The vertical shaft impact crusher has a complicated structure and expensive parts. If the influence of the structural and production parameters of the rotor on the wear of the split cone is explored by experiment, each replacement of parts will waste time and increase costs. At the same time, environmental conditions and other factors also have an impact on the experimental results. Due to the low cost of the simulation and no need to consider the influence of environmental factors, this paper adopts the method of simulation to explore the influence of different factors on the wear of the split cone.

2.1. Rotor Model. A structural schematic of the vertical shaft impact crusher is shown in Figure 1. It is mainly composed of spindle assembly, motor, rotor, crushing cavity, feed hopper, and control system. The three-dimensional model of the rotor is shown in Figure 2, which is driven by the spindle of the vertical shaft impact crusher. The rotor is mainly composed of a feeding ring, split cone, guide plate, and upper and lower wear-resistant plates. It is located in the center of the rotor. After the particles are fed by the feeding device, they enter into the rotor through the feeding ring and the split cone distributes the particles evenly on both sides of the guide plate. The particles are accelerated, collided, and ground inside the rotor and the rotor is left under the action of centrifugal force. Then they collide with the lining plate and material layer of the crushing cavity to achieve the crushing [6].

When the particles enter into the rotor from the feeding ring and impact on the split cone with a certain kinetic energy, it will certainly lead to wear on the surface of the split cone. The particles are uniformly distributed on both sides of the guide plate by the split cone and they are continuously accelerated and thrown out after contact with the guide plate. At this time, the content of particles at the entrance of

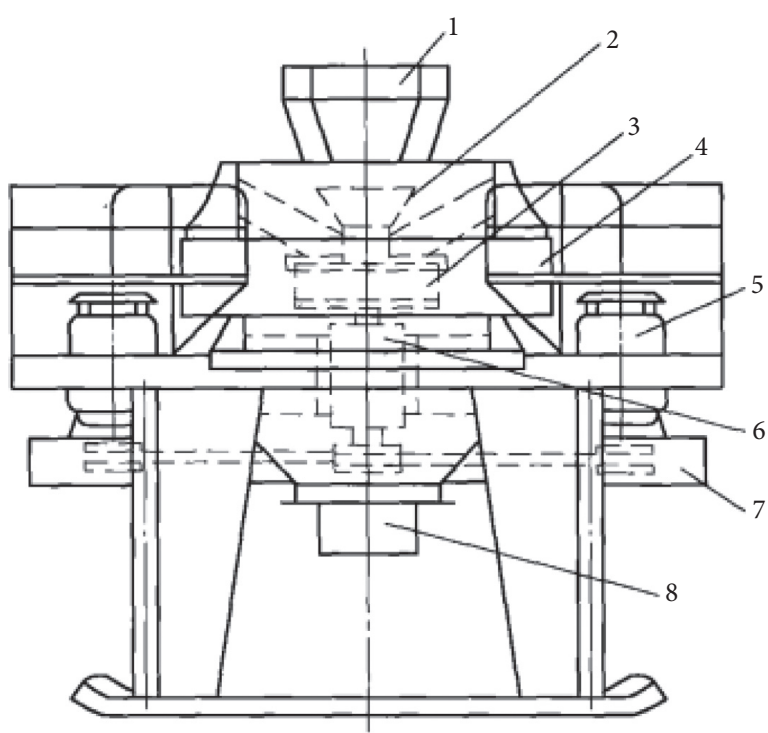

FIgURE 1: The vertical shaft impact crusher. 1-feeder; 2-splitter; 3rotor; 4-crushing cavity; 5-motor; 6- spindle assembly; 7-rack; 8hopper.

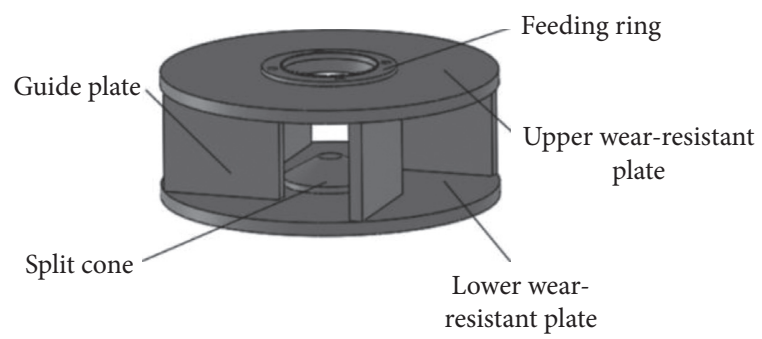

FIgURE 2: Three-dimensional model of the rotor.

the guide plates is relatively small. As the particles continue to enter the rotor, the area with more particles will inevitably flow to the area with fewer particles. During the flow of particles, the relative sliding between the particles and the split cone will also cause wear on the surface of the split cone. When the installation angle of the guide plate is $\theta$ and the installation number is 4 , the structural parameters of the rotor are shown in Figure 3.

2.2. Wear Model of the Split Cone. Based on Hertz contact theory and Archard wear design calculation model, a calculation method suitable for the wear of the split cone was established $[17,18]$. The wear value of the split cone was estimated so that it could be used in the subsequent simulation analysis.

In the process of simulation, the shape of the particles was spherical and the contact between the particle and the surface of the split cone was calculated according to Hertz contact theory. Because the contact area between the spherical particle and the surface of the split cone was small, large local stress that caused plastic deformation or even ruptures on the surface of the split cone was generated in the contact area. The Hertz contact model is shown in Figure 4.

According to Hertz Contact Theory, 


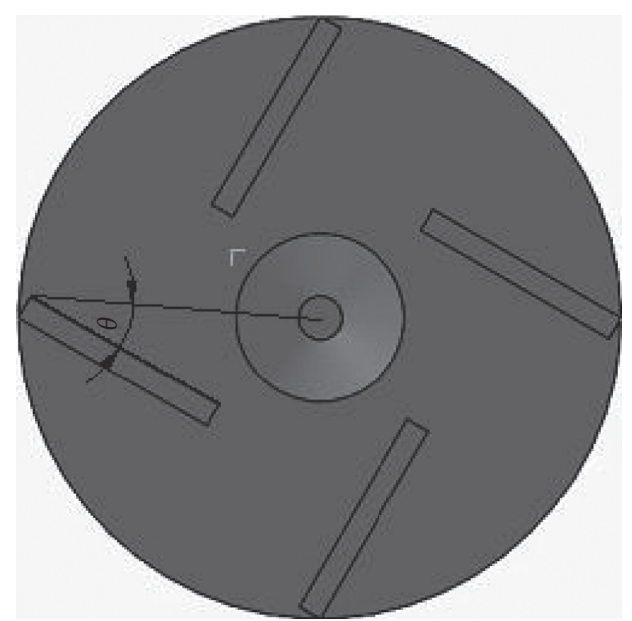

FIGURE 3: The structural parameters of the rotor.

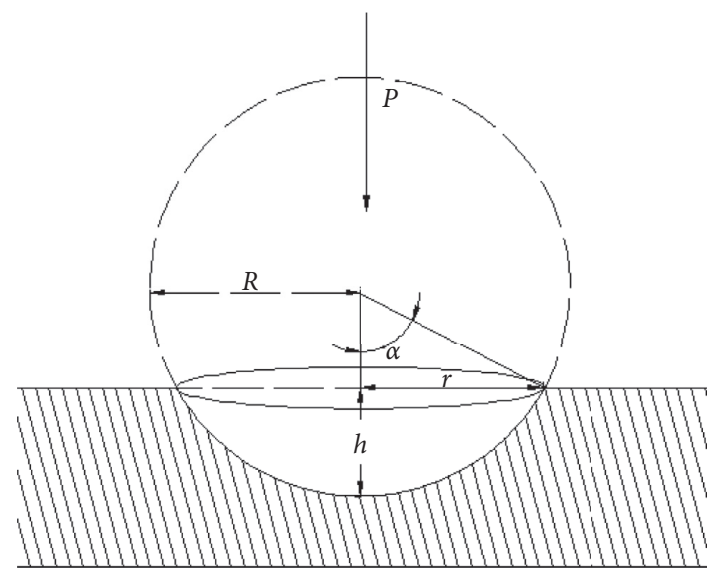

Figure 4: Hertz contact model.

$$
r=\sqrt{2 R h}
$$

where $r$ is the circular radius of the horizontal section of the particle pressed into the surface $(\mathrm{mm}) ; R$ is the radius of the particle $(\mathrm{mm}) ; h$ is the maximum indentation depth $(\mathrm{mm})$.

As shown in Figure 5, assuming that a particle with a mass of $m$ impacts the surface of the split cone at velocity $v$, it will produce a contact load with the size of $P$, which is related to the hardness of the contact surface. The normal component of the contact load will cause an indentation on the surface of the split cone, which can be calculated by the following formula:

$$
\begin{gathered}
P_{n}=H A(h), \\
A(h)=\pi r^{2},
\end{gathered}
$$

where $P_{n}$ is the normal contact load $(N), A(h)$ is the horizontal cross-sectional circle area of the particle pressed into the surface of the split cone $\left(\mathrm{mm}^{2}\right)$, and it is a function related to the maximum indentation depth $h$.

Assuming that the normal kinetic energy of the particle impacting the surface of the split cone is all used to make the surface of the split cone rupture and deform, then,

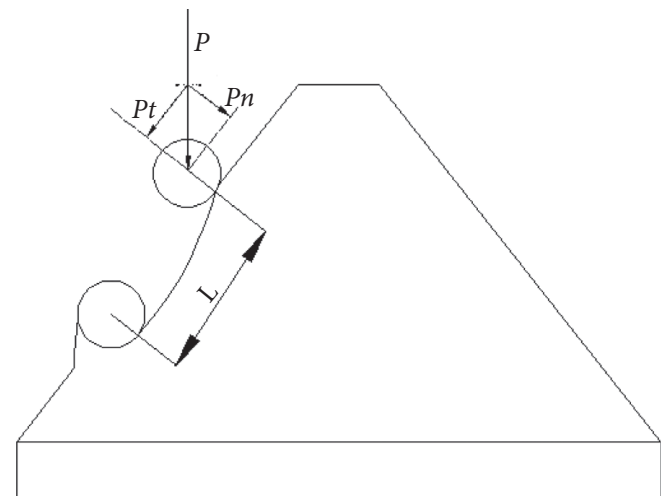

FIgURE 5: Schematic diagram of the particle impact split cone.

$$
\begin{aligned}
& E_{n}=\frac{1}{2} m v_{n}^{2}, \\
& \omega_{n}=\int_{0}^{h} P_{n} \mathrm{~d} h=\pi R h^{2} H, \\
& \omega_{n}=E_{n},
\end{aligned}
$$

where $E_{n}$ is the normal kinetic energy of the particle impacting the surface of the split cone, $v_{n}$ is the normal velocity when the particle is in contact with the surface of the split cone, $\omega_{n}$ is the work done by the normal load.

The maximum indentation depth of the particle can be obtained from formulas (2)-(4):

$$
h=\frac{P_{n}}{2 \pi R H} \text {. }
$$

The relationship between normal load and normal kinetic energy can be obtained from formulas (2)-(5):

$$
P_{n}=\sqrt{4 \pi R H E_{n}} .
$$

It can be seen from Figure 5 that the particle not only has a normal effect on the surface of the split cone but also has a tangential effect.

The tangential displacement of the particle with a certain tangential velocity on the surface of the split cone can be calculated using

$$
L=v_{t} \cdot t
$$

where $L$ is the tangential displacement of the particle, $v_{t}$ is the tangential velocity when the particle is in contact with the surface of the split cone, $t$ is the contact time between the particle and the surface of the split cone.

The cross-sectional area of the particle pressed into the surface of the split cone can be calculated using

$$
\begin{aligned}
& S=\alpha R^{2}-r(R-h), \\
& \alpha=\arcsin \frac{r}{R},
\end{aligned}
$$

where $S$ is the cross-sectional area. 
After the surface of the split cone is impacted by the particle, the volume of the material lost on the surface of the split cone can be calculated using

$$
V=S \cdot L
$$

where $V$ is the volume of material lost on the surface of the split cone.

Equations (1) to (12) were programmed by programming software and coupled with EDEM through the API interface of EDEM. The wear model was defined as the Hertz-Mindlin with Custom Wear model, which was used to estimate the wear value of the split cone.

\section{Wear Model Verification}

3.1. Setting of Simulation Parameters. In the simulation software EDEM, the material of the particles was defined as limestone and the material of the rotor was defined as steel. In EDEM, the Hertz-Mindlin (no-slip) contact model was used between particles [19] and the Hertz-Mindlin with Custom Wear model was used between particles and rotor.

The material properties of the particle and rotor are shown in Table 1. The contact parameters between the particle and rotor are shown in Table 2.

In the actual sand-making process, the shape and size of particles are complex and changeable, and the gradation of particles also has a certain degree of continuity. In the process of simulation, the shape of the particles was simplified and set to be spherical. To improve the authenticity of the simulation, the particles with particle sizes of $8 \mathrm{~mm}$, $10 \mathrm{~mm}$, and $12 \mathrm{~mm}$ were selected. In the simulation, the three kinds of particles were mixed in proportions of $30 \%$, $40 \%$, and $30 \%$, respectively. Finally, the simulation time was set to $10 \mathrm{~s}$.

3.2. Physical Experiment Validation. To verify the effectiveness of the wear model and ensure the rationality of the subsequent simulation results, the physical experiment was completed jointly with the technical personnel of the relevant cooperative enterprise. The material properties of the particles and rotor used in the physical experiment, the particle size of the particles and the mass proportion of each particle size were the same as the simulation experiment. The amount of feed was also the same as the simulation test, which was set to $10 \mathrm{t} / \mathrm{h}$.

The parameters of the rotor are as follows: the installation angle of the guide plate is $30^{\circ}$, the installation number is 3 , the rotor speed is $2800 \mathrm{r} / \mathrm{min}$, and the feed height is $80 \mathrm{~mm}$.

The physical diagram of the split cone is shown in Figure 6(a). As shown in Figure 6(b), the split cone is divided into 10 equal parts from the inside to the outside of the shaft hole, and the wear values of each area are recorded, respectively.

After the rotor has been working for a period of time, the split cone was removed and the wear value of the split cone was measured by the 3D scanner and related software [20]. After three physical experiments, the average wear values of
TABLE 1: Material property table.

\begin{tabular}{lccc}
\hline Materials & Poisson's ratio & $\begin{array}{c}\text { Density } \\
\left(\mathrm{kg} / \mathrm{m}^{3}\right)\end{array}$ & Shear modulus (pa) \\
\hline Limestone & 0.25 & 2640 & $2.09 \times 10^{8}$ \\
Steel & 0.30 & 7800 & $7 \times 10^{8}$ \\
\hline
\end{tabular}

TABLE 2: Contact parameter table.

\begin{tabular}{lccc}
\hline $\begin{array}{l}\text { Contact } \\
\text { materials }\end{array}$ & $\begin{array}{c}\text { Recovery } \\
\text { coefficient }\end{array}$ & $\begin{array}{c}\text { Static coefficient of } \\
\text { friction }\end{array}$ & $\begin{array}{c}\text { Scroll the } \\
\text { coefficient of } \\
\text { friction }\end{array}$ \\
\hline $\begin{array}{l}\text { Limestone } \\
\text { and limestone }\end{array}$ & 0.207 & 0.77 & 0.1 \\
$\begin{array}{l}\text { Limestone } \\
\text { and steel }\end{array}$ & 0.557 & 0.61 & 0.07 \\
\hline
\end{tabular}

each area of the split cone were the experimental wear value. The experimental wear value was defined $V$. The simulated wear values of each area of the split cone were obtained by using the postprocessing function of EDEM. The simulation wear value was defined $Y$.

The experimental wear values are compared with the simulation wear values and the result is shown in Figure 7.

It can be seen from Figure 7 that the curve of the physical experiment is very similar to the curve of the simulation experiment. To compare the difference more intuitively, the simulation data are processed as follows.

Firstly, based on the physical experimental value of area number $i$, it is assumed that there is no deviation between the simulation wear value and the experimental wear value in area number $i$. Secondly, the simulation wear values of other areas are calculated by using formulas (13) and (14). Finally, formula (15) is used to calculate the relative error of wear between the simulation wear value and the experimental wear value in each area to estimate the deviation between the simulation wear value and the experimental wear value.

$$
\begin{aligned}
\varepsilon_{i} & =\frac{Y_{i}}{V_{i}}, \\
y_{j} & =\frac{Y_{j}}{\varepsilon_{i}}, \\
E & =\frac{y_{j}-V_{j}}{V_{j}} \times 100 \%,
\end{aligned}
$$

where $Y_{i}$ and $V_{i}$ are, respectively, the simulation wear value and experimental wear value of area number $i, Y_{j}$ and $V_{j}$ are, respectively, the simulation value and experimental value of area number $j$ when the experimental value of area number $i$ is taken as the benchmark, $i$ and $j$ are integers between 1 and $10, E$ is the relative error.

Based on the experimental values of area numbers 2, 4, 6, 8 , and 10 , in turn, the relative errors of each area are calculated and the results are shown in Figure 8 . As can be known from Figure 8 that the relative errors between the simulation wear value and the experimental wear value are within $10 \%$, so it can be considered that the wear model can effectively predict the wear value of the split cone. 


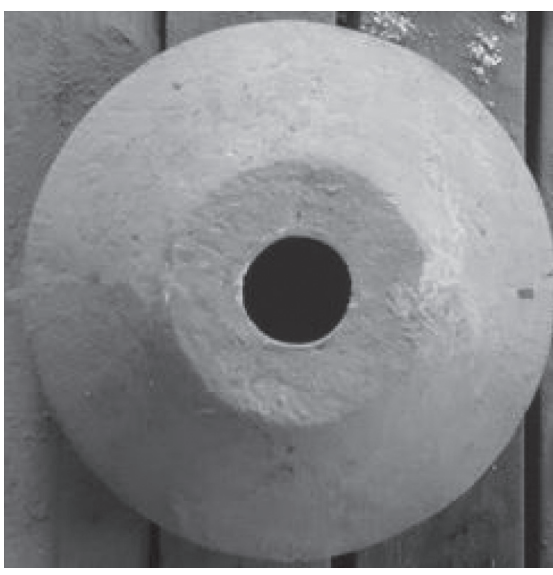

(a)

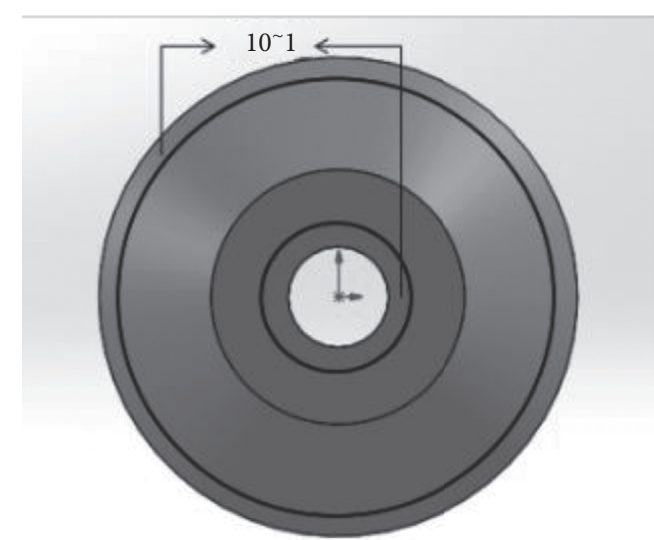

(b)

Figure 6: Physical drawing (a) and simulation model (b) of the split cone.

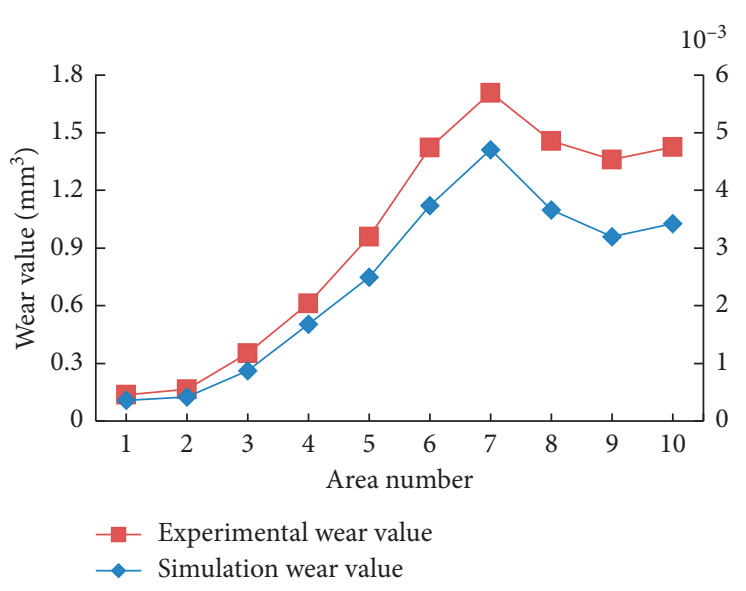

FIGURE 7: Simulation and physical experiment results.

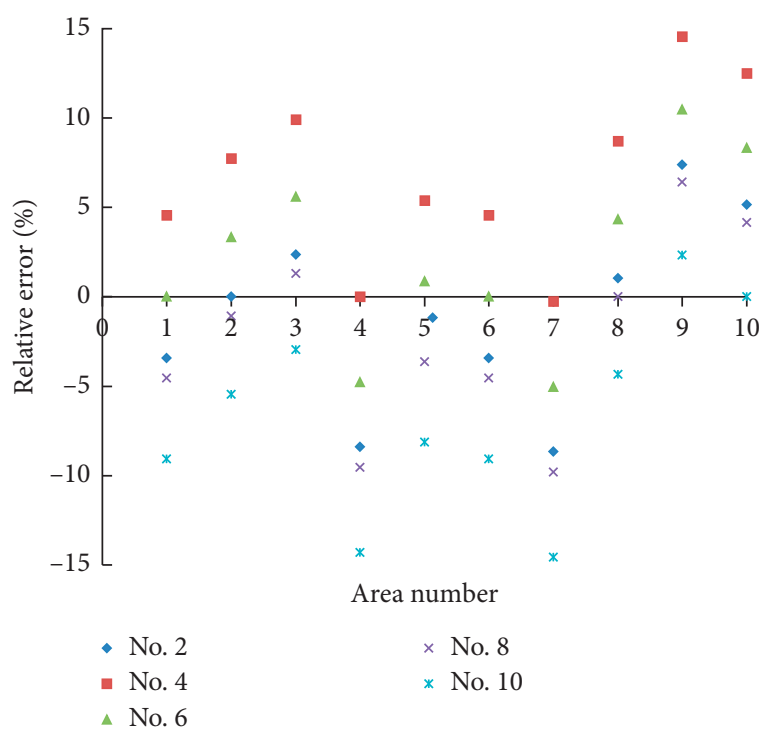

FIgURE 8: The relative error.

\section{Test Design}

In actual engineering, the wear of the split cone is not only caused by a single factor but the result of the joint action of many factors. Therefore, it is not accurate to consider only a single factor in wear analysis. With the increase of the number of factors, the number of trials is also increasing sharply and the results are not satisfactory. In terms of time, material, and financial resources, the number of trials should be reduced as much as possible. The orthogonal design has the advantages of "uniform dispersion" and "neat comparability" [21]. It can also greatly reduce the number of tests, so it meets the requirements of use.

4.1. The Level of Factors for Orthogonal Design. The structural parameters of the rotor (the installation angle and number of the guide plate) and the production parameters (the rotor speed and the feed height) were selected as the research factors. Considering the crushing effect of particles, the installation method of the guide plate adopted the forward guide plate [1].

Through the communication with the technical personnel of the relevant cooperative enterprises, the selection of factor level is shown in Table 3.

4.2. Assessment Indicators. The split cone is divided into $n$ grids and the wear value of the $i$-th grid is defined as $u_{i}$. The total wear value of the split cone can be calculated using

$$
U=\sum_{i=1}^{n} u_{i}
$$

where $U$ is the total wear value of the split cone, $i$ is a positive integer.

The relative wear degree of each mesh can be calculated using 
TABLE 3: Level of factors table.

\begin{tabular}{lcccc}
\hline Factors & Installation angle $\theta\left(^{\circ}\right)$ & Installation number $m$ & Speed $n(\mathrm{r} / \mathrm{min})$ & Feed height $h(\mathrm{~mm})$ \\
\hline Level 1 & 25 & 3 & 2000 & 40 \\
Level 2 & 30 & 4 & 2400 & 80 \\
Level 3 & 35 & 5 & 2800 & 120 \\
Level 4 & 40 & 6 & 3200 & 160 \\
\hline
\end{tabular}

$$
s_{i}=\frac{u_{i}}{U}
$$

where $s_{i}$ is the relative wear degree of each mesh.

The wear uniformity of the split cone can be calculated using

$$
\sigma=\sqrt{\frac{1}{n} \sum_{i=1}^{n}\left(s_{i}-\bar{s}\right)^{2}},
$$

where $\bar{s}$ is the average of the relative wear degree; $\sigma$ is the wear uniformity of split cone.

Considering that the particles should have enough kinetic energy when they leave the rotor, the average velocity of the particles thrown away from the rotor, the total wear value, and the wear uniformity of the split cone were selected as the assessment indicators.

4.3. Scheme Design and Test Results. According to the factor level Table, the orthogonal test Table of $L_{16}\left(4^{4}\right)$ was established. After the simulation test, the simulation results of each assessment indicator were sorted out. The orthogonal test scheme and test results are shown in Table 4.

\section{Results Analysis}

The range analysis method was used to analyze the influence order of each factor on the assessment indicators and the influence law of each factor on the total wear value and wear uniformity of the split cone. Through the regression analysis of the test results, the optimal parameter combination of various factors was obtained to improve the wear resistance of the split cone.

5.1. The Range Analysis Method. If the average velocity of particles thrown away from the rotor is larger and the total wear value and wear uniformity of the cone are smaller, the wear resistance of the split cone is better.

The result of the range analysis is shown in Table 5, where $\overline{K_{i}}$ is the average value of the sum of the data corresponding to each factor at the level of $i, R$ is the range of the comprehensive average value of each factor at each level. If the $R$ value is larger, it indicates that the factor has a greater impact on the assessment indicator.

The results show that the influence order of various factors on the total wear value of the split cone is the rotor speed, installation angle, feed height, and number of installations, respectively; the influence order of various factors on the uniformity of the wear of the split cone is the installation angle, rotor speed, number of installations and feed height, respectively; the influence order on the average velocity of particles thrown away from the rotor is the rotor speed, installation angle, number of installations, and feed height, respectively.

To more intuitively analyze the influence of various factors on the wear of the split cone, as shown in Figure 9, the curves of the relationship between the factors and the total wear value and wear uniformity of the split cone are drawn according to Table 5 .

It can be seen from Figure 9(a) that the total wear value and wear uniformity of the split cone decrease with the increase of the installation angle of the guide plate. By comparing the total mass of particles inside the rotor when the simulation time is $6 \mathrm{~s}$ when the installation angle of the guide plate is $25^{\circ}$, the total mass of particles inside the rotor is the most. It is not difficult to imagine that the more particles, the more times of contact with the surface of the split cone per unit time, so the total wear value of the split cone is also greater. When the installation angle of the guide plate increases from $25^{\circ}$ to $40^{\circ}$, the total wear value of the split cone decreases because the total mass of particles inside the rotor continues to decrease. If the installation angle of the guide plate is large, the particles will have a large distribution space inside the rotor before they come into contact with the guide plate. It means that the particles are more evenly distributed in the rotor and the wear uniformity of the split cone is smaller.

It can be seen from Figure 9(b) that the total wear value and wear uniformity of the split cone do not change monotonously with the increase in the number of installations of the guide plate. The total wear value of the split cone is the smallest when the installation angle of the guide plate is 4 . When the installation angle of the guide plate is 3 , the wear uniformity of the split cone is the best. The reason is that the number of installations of the guide plate has a certain influence on the particle flow inside the rotor.

It can be seen from Figure 9(c) that the total wear value and wear uniformity of the split cone increase with the increase of the rotor speed. On the one hand, if the rotor speed is very high, the relative speed between the surface of the split cone and the particle is very high at the first contact. It will have a great impact on the surface of the split cone. On the other hand, the particles inside the rotor are continuously accelerated. The particles inside the rotor are continuously accelerated. The kinetic energy of the accelerated particles increases with the increase of rotor speed. When the surface of the split cone is in contact with the particles again, the impact of the particles is higher. In conclusion, when the rotor speed is large, the total wear value and wear uniformity of the split cone are also large. 
TABLE 4: Test scheme and results.

\begin{tabular}{|c|c|c|c|c|c|c|c|}
\hline \multirow[b]{2}{*}{ Test No } & \multicolumn{4}{|c|}{ Test factors } & \multirow[b]{2}{*}{ Total wear value $U / 10^{-3}\left(\mathrm{~mm}^{3}\right)$} & \multirow[b]{2}{*}{ Wear uniformity $\sigma$} & \multirow[b]{2}{*}{$\begin{array}{c}\text { Average velocity } \\
\qquad V(\mathrm{~m} / \mathrm{s})\end{array}$} \\
\hline & Installation angle $\theta$ & $\begin{array}{c}\text { Number of } \\
\text { installation } m\end{array}$ & Rotor speed $n$ & Feed height $h$ & & & \\
\hline 1 & 25 & 3 & 2000 & 40 & 0.1507 & 0.2327 & 79.35 \\
\hline 2 & 25 & 4 & 2400 & 80 & 0.2239 & 0.4058 & 98.39 \\
\hline 3 & 25 & 5 & 2800 & 120 & 0.3403 & 0.5604 & 116.58 \\
\hline 4 & 25 & 6 & 3200 & 160 & 0.4383 & 0.6082 & 128.54 \\
\hline 5 & 30 & 3 & 2400 & 120 & 0.2254 & 0.2213 & 87.64 \\
\hline 6 & 30 & 4 & 2000 & 160 & 0.1798 & 0.2093 & 75.53 \\
\hline 7 & 30 & 5 & 3200 & 40 & 0.3143 & 0.4123 & 121.92 \\
\hline 8 & 30 & 6 & 2800 & 80 & 0.2808 & 0.3014 & 108.81 \\
\hline 9 & 35 & 3 & 2800 & 160 & 0.2494 & 0.2883 & 87.68 \\
\hline 10 & 35 & 4 & 3200 & 120 & 0.2668 & 0.3559 & 120.68 \\
\hline 11 & 35 & 5 & 2000 & 80 & 0.1361 & 0.1807 & 77.24 \\
\hline 12 & 35 & 6 & 2400 & 40 & 0.1643 & 0.2090 & 87.19 \\
\hline 13 & 40 & 3 & 3200 & 80 & 0.2343 & 0.2665 & 86.37 \\
\hline 14 & 40 & 4 & 2800 & 40 & 0.1432 & 0.1590 & 94.98 \\
\hline 15 & 40 & 5 & 2400 & 160 & 0.1962 & 0.3175 & 76.53 \\
\hline 16 & 40 & 6 & 2000 & 120 & 0.1534 & 0.1648 & 65.98 \\
\hline
\end{tabular}

TABLE 5: Range analysis table.

\begin{tabular}{|c|c|c|c|c|c|}
\hline & & Installation angle & Number of installations & Rotor speed & Feed height \\
\hline \multirow{5}{*}{ Total wear value } & $\overline{\overline{K_{1}}}$ & 0.2883 & 0.2150 & 0.1550 & 0.1931 \\
\hline & $\overline{K_{2}}$ & 0.2501 & 0.2034 & 0.2025 & 0.2188 \\
\hline & $\frac{-2}{K_{3}}$ & 0.2042 & 0.2467 & 0.2534 & 0.2465 \\
\hline & $\overline{K_{4}}$ & 0.1818 & 0.2592 & 0.3134 & 0.2659 \\
\hline & $R$ & 0.1065 & 0.0558 & 0.1584 & 0.0728 \\
\hline \multirow{5}{*}{ Wear uniformity } & $\overline{K_{1}}$ & 0.4518 & 0.2522 & 0.1969 & 0.2533 \\
\hline & $\underline{\overline{K_{2}}}$ & 0.2861 & 0.2825 & 0.2884 & 0.2886 \\
\hline & $\overline{K_{3}}$ & 0.2585 & 0.3677 & 0.3273 & 0.3256 \\
\hline & $\overline{K_{4}}$ & 0.2270 & 0.3209 & 0.4107 & 0.3558 \\
\hline & $R^{4}$ & 0.2248 & 0.1155 & 0.2139 & 0.1026 \\
\hline \multirow{5}{*}{ Average } & $\overline{\overline{K_{1}}}$ & 105.72 & 85.26 & 74.53 & 95.86 \\
\hline & $\overline{K_{2}}$ & 98.48 & 97.40 & 87.44 & 92.70 \\
\hline & $\overline{K_{3}}$ & 93.20 & 98.07 & 102.01 & 97.72 \\
\hline & $\overline{K_{4}}$ & 80.79 & 97.63 & 114.38 & 92.07 \\
\hline & $R$ & 24.75 & 12.81 & 39.85 & 5.65 \\
\hline
\end{tabular}

It can be seen from Figure 9(d) that the total wear value and wear uniformity of the split cone increase with the increase of the feed height. The particles freely fall into the rotor. When the feed height is very high, the kinetic energy of the particles is greater when they contact the surface of the split cone surface. It will have a great impact on the surface of the split cone. Thus, the total wear value and wear uniformity of the split cone increase with the increase of the feed height.

5.2. Regression Analysis. Multiple stepwise regression analysis, developed on the basis of multiple linear regression analysis, can pick out the independent variables which have a significant influence on the dependent variables from a number of independent variables. These significant independent variables are used to establish a linear regression model.

In this paper, the data processing software of SPSS is used for multiple stepwise regression analysis of test data
$[22,23]$. The regression equations of total wear value $Y_{U}$, wear uniformity $Y_{\sigma}$, and average velocity $Y_{V}$ are obtained as follows:

$$
\begin{aligned}
Y_{U}= & -0.0142-7.31 \times 10^{-3} x_{1}+0.0176 x_{2}+1.32 \times 10^{-4} x_{3} \\
& +6.15 \times 10^{-4} x_{4} \\
Y_{\sigma}= & 0.103-0.014 x_{1}+0.0291 x_{2}+1.70 \times 10^{-4} x_{3} \\
& +8.62 \times 10^{-4} x_{4} \\
Y_{V}= & 42.09-1.59 x_{1}+3.78 x_{2}+0.034 x_{3}
\end{aligned}
$$

where $x_{1}$ is the installation angle of the guide plate, $x_{2}$ is the number of installations for the guide plate, $x_{3}$ is the rotor speed, $x_{4}$ is the feed height. 


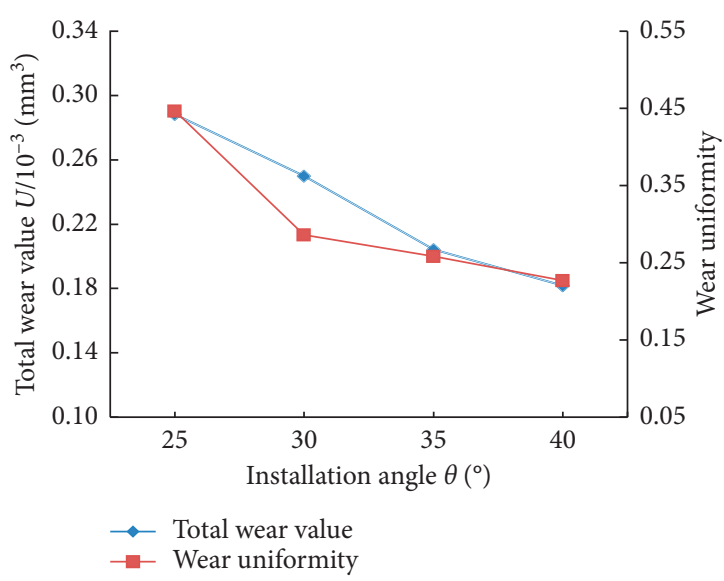

(a)

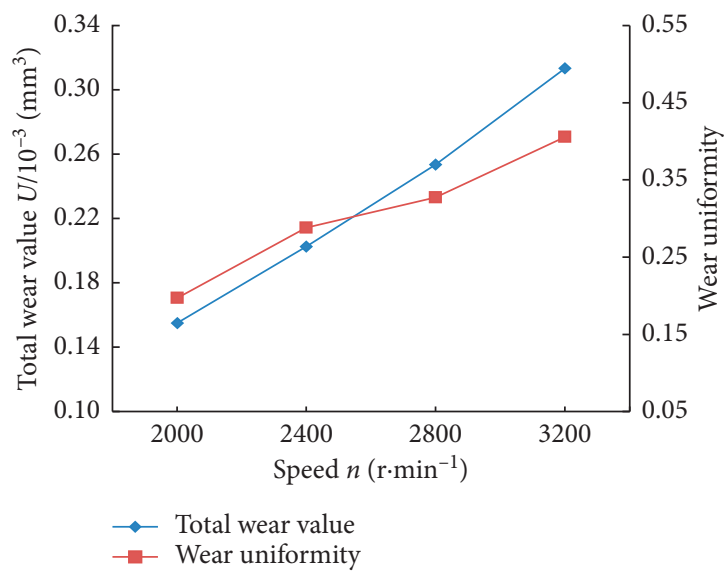

(c)

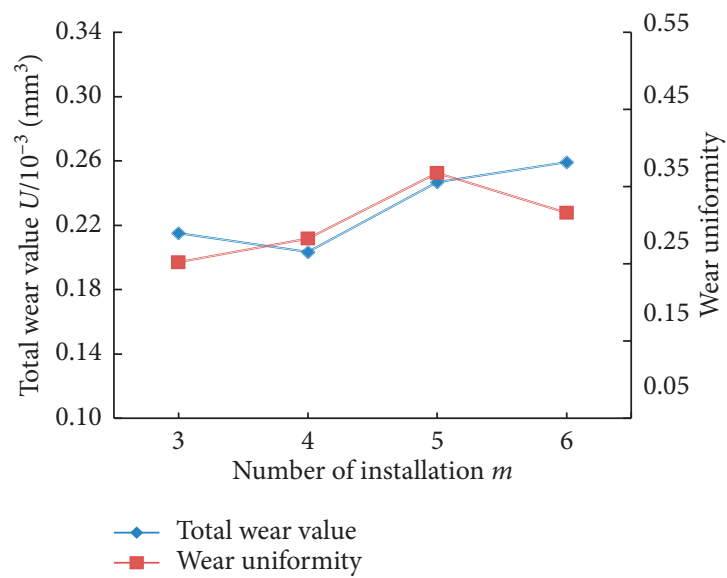

(b)

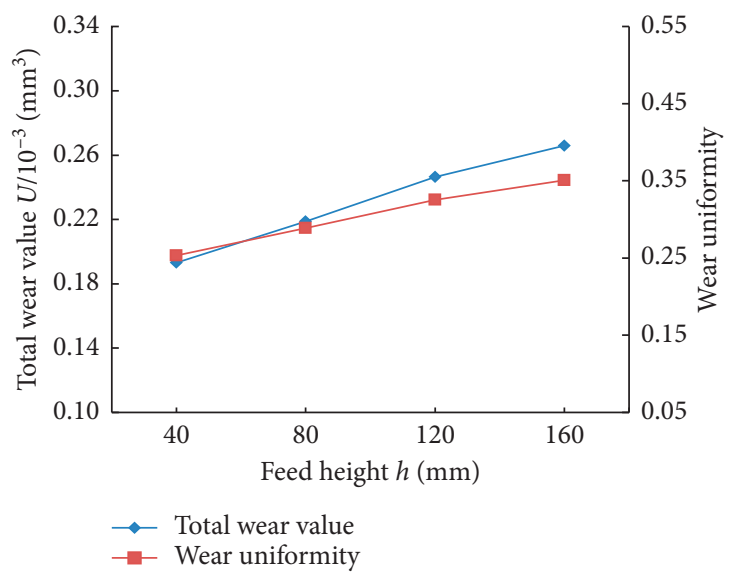

(d)

Figure 9: Wear of the split cone under different levels of each factor. (a) Installation angle. (b) Number of installation. (c) Speed. (d) Feed height.

The total correlation coefficient is an indicator used to describe the linear correlation degree of the regression equation. The closer its value is to 1 , the greater the linear correlation between independent variables and dependent variables.

The correlation coefficients of the regression equations of (19)-(21) are $0.985,0.926$, and 0.964 , respectively, which are all close to 1 , indicating that the established stepwise regression models for total wear value, wear uniformity, and average velocity have high accuracy.

The variance analysis of the regression equations is shown in Table 6. The significant level is $\alpha=0.05$. If $F$ value is greater than the critical value, the regression equation is significant. Otherwise, it is not significant. As is apparent from the table, $F_{U}=89.082 \gg F_{0.05}(4,11), F_{\sigma}=16.576 \gg F_{0.05}(4,11), F_{V}=$ $50.607 \gg F_{0.05}(3,12)$ indicates that the established regression equations are highly significant. In other words, the rotor speed, feed height, installation angle, and the number of guide plates have significant influence on the average velocity of the particles thrown away from the rotor, the total wear value, and the wear uniformity of the split cone in the sand-making process.
5.3. Parameter Optimization. According to the established regression models, the four parameters of installation angle of the guide plate $\left(x_{1}\right)$, the number of installations of guide plate $\left(x_{2}\right)$, rotor speed $\left(x_{3}\right)$, and feed height $\left(x_{4}\right)$ are the design variables, the regression equations of (19) and (20) are the objective function and the production parameters and structural parameters are constrained, the mathematical model of wear optimization is established to improve the wear resistance of the split cone.

The resulting mathematical model of wear optimization is as follows:

$$
\begin{aligned}
& \vec{V}-\min Y(\vec{X})=\min \left[Y_{U}(\vec{X}) Y_{\sigma}(\vec{X})\right]^{T} \\
& \vec{X} \in \vec{D} \subset \vec{R}^{n} \\
& \vec{D}: Y_{V}(\vec{X}) \geq 85 \\
& 25^{\circ} \leq x_{1} \leq 40^{\circ} \\
& \left.\begin{array}{l}
3 \leq x_{2} \leq 6 \\
2000 \leq x_{3} \leq 3200 \\
40 \leq x_{4} \leq 160
\end{array}\right\}
\end{aligned}
$$


TABLE 6: Anova table of regression models.

\begin{tabular}{|c|c|c|c|c|c|c|}
\hline & The source of the variance & The sum of squares & Freedom & Average variance & $F$ value & The critical value \\
\hline \multirow{3}{*}{ Total wear value } & Regression & 0.100 & 4 & 0.025 & \multirow{3}{*}{89.082} & \multirow{3}{*}{$F_{0.05}(4,11)=3.36$} \\
\hline & Residuals & 0.003 & 11 & $2.82 \times 10^{-4}$ & & \\
\hline & Total & 0.103 & 15 & & & \\
\hline \multirow{3}{*}{ Wear uniformity } & Regression & 0.232 & 4 & 0.058 & \multirow{3}{*}{16.576} & \multirow{3}{*}{$F_{0.05}(4,11)=3.36$} \\
\hline & Residuals & 0.038 & 11 & 0.003 & & \\
\hline & Total & 0.270 & 15 & & & \\
\hline \multirow{3}{*}{ Average velocity } & Regression & 5148.734 & 3 & 1716.245 & \multirow{3}{*}{52.607} & \multirow{3}{*}{$F_{0.05}(3,12)=3.49$} \\
\hline & Residuals & 391.485 & 12 & 32.624 & & \\
\hline & Total & 5540.218 & 15 & & & \\
\hline
\end{tabular}

The hierarchical sequence method may be interrupted during the solution process, which makes it impossible to continue the solution process. The tolerance hierarchical sequence method can give a width capacity to the optimal value of each objective function in advance, thus avoiding the interruption of the calculation process. Therefore, this paper adopts the tolerant hierarchical sequence method to find the optimal solution of the objective function [24].

The first level:

$$
\min Y_{U}(\vec{X})=M_{1}
$$

Take the tolerance value $\gamma_{1}=0.05$. Then, the second level:

$$
\left\{\begin{array}{l}
\min Y_{\sigma}(\vec{X}) \\
\vec{X} \in \overrightarrow{D_{1}}=\left\{\vec{X} \mid Y_{U}(\vec{X})<M_{1}+0.05, \vec{X} \in \vec{D}\right\} .
\end{array}\right.
$$

According to the solution algorithm flow of the tolerant hierarchical sequence method, the solution program is written.

Finally, the optimal combination of the parameters was obtained: the installation angle of the guide plate was $40^{\circ}$, the number of installations of the guide plate was 3 , the rotor speed was $2800 \mathrm{r} / \mathrm{min}$, and the feed height was $40 \mathrm{~mm}$. The simulation test was carried out under the optimal parameter combination, and the simulation results were as follows: the total wear value of the split cone was 0.1483 , the wear uniformity was 0.1425 , and the average velocity of the particles thrown away from the rotor was 90.67 .

\section{Conclusions}

The purpose of this work is to determine the optimal parameters of the rotor through simulation and experiments so as to reduce the wear of the split cone. Based on Hertz contact theory and Archard wear design calculation model, the wear model of the split cone was established to predict the wear value of the split cone. The experiments were designed according to the $L_{16}\left(4^{4}\right)$ orthogonal array. In addition, the influence of rotor structure parameters and production parameters on the wear of the split cone was determined by the range analysis method. However, multiple linear regression analysis was performed to estimate experimental results. The results are summarized as follows:
(1) The simulation and experimental results showed that the curve of the physical experiment was very similar to the curve of the simulation experiment. At the same time, the relative error between the simulation wear value and the experimental wear value was within $10 \%$ in five different regions of the split cone, which indicated that the wear model could effectively predict the wear value of the split cone.

(2) The analysis of the results of the orthogonal test showed that the total wear value and wear uniformity of the split cone did not change with the increase of the number of installations of the guide plate. The total wear value and wear uniformity of the split cone decreased with the increase of the installation angle of the guide plate, so a larger installation angle would be selected. The total wear value and wear uniformity of the split cone increased with the increase of rotor speed and feed height, so lower rotor speed and feed height would be selected.

(3) The results of the orthogonal experiment were analyzed by multiple linear regression, and the regression equations were all significant. The correlation coefficients of the regression equations for the total wear value, wear uniformity, and average velocity were $0.985,0.926$, and 0.964 , respectively.

(4) With regression equations as the objective functions, production parameters, and structural parameters as constraints, a mathematical model for wear optimization was established. The optimal solution of the mathematical model of wear optimization was calculated by using the tolerance layering sequence method. The optimal parameter combination of each factor was obtained as follows: the installation angle of the guide plate was $40^{\circ}$, the number of the guide plate was 3, the rotor speed was $2800 \mathrm{r} / \mathrm{min}$, and the feed height was $40 \mathrm{~mm}$.

\section{Data Availability}

No data were used to support the findings of this study.

\section{Disclosure}

Jie Yang and Feifei Feng are co-first authors. 


\section{Conflicts of Interest}

The authors declare that there are no conflicts of interest regarding the publication of this paper.

\section{Authors' Contributions}

Jie Yang and Feifei Feng contributed equally to this work

\section{Acknowledgments}

The project was supported by the National Natural Science Foundation of China: Research on Enterprise Resource Location Optimization Based on the Internet of Things (71371172) and Henan 2018 Science and Technology Project: Key Technologies and Development of Big Databased Intelligent Control System and Production Management Platform for Mixing Plant (182102210060).

\section{References}

[1] B.-x. Lang, Crusher, Metallurgical Industry Press, Beijing, China, 2008.

[2] B. Xu, "Quality discussion of artificial sand in terms of sandmaking machine," Shandong Communications Technology, vol. 325, no. 8, pp. 99-101, 2016.

[3] C. Gui, "The Archard design calculation model and its application methods," Lubrication Engineering, vol. 77, no. 1, pp. 12-21, 1990.

[4] H. S. Grewal, H. Singh, and A. Agrawal, "A phenomenological model for slurry erosion prediction of thermal spray coatings," Tribology Letters, vol. 56, no. 1, pp. 119-132, 2014.

[5] I. Finnie, "Some observations on the erosion of ductile metals," Wear, vol. 19, no. 72, pp. 81-90, 1972.

[6] I. Finnie, "Some reflections on the past and future of erosion," Wear, vol. 186-187, pp. 1-10, 1995.

[7] H. Guang, Z. Cai, and Z. Chen, "Impact fretting wear behaviour of thin-walled tubes with different support structures," Journal of Vibration and Shock, vol. 36, no. 21, pp. 65-71, 2017.

[8] Anonymous, "Thyssenkrupp touts new Kubria cone crusher," Rock Products, vol. 119, no. 11, 2016.

[9] T. Yin, H. Fang, and J. Yang, "Simulation and analysis on wear of accelerating board of vertical-shaft impact crusher," Mining Machinery, vol. 45, no. 6, pp. 37-42, 2017.

[10] P. Liu and S. Yang, "Wear model of disc hob cutter rings based on abrasive wear," China Mechanical Engineering, vol. 30, no. 15, pp. 1782-1789, 2019.

[11] L. Xu, K. Luo, and Y. Zhao, "Effect of particle size on liner wear in semi-autogenous mill," Journal of Zhejiang University (Engineering Science), vol. 53, no. 12, pp. 2255-2263, 2019.

[12] C. Zhang, L. Zhao, and Y. Kang, "Structure optimization of rotor of vertical shaft impact crusher based on discrete element method," Machine Design \& Research, vol. 34, no. 6, pp. 85-88, 2018.

[13] L. Lu and L. Houzhiqiang, "Research into the wear mechanism of vertical shaft impactcrusher rotor based on DEM," China Mining Industry, vol. 25, no. s2, pp. 312-316, 2016.

[14] J. Terva, K. Valtonen, P. Siitonen, and V.-T. Kuokkala, "Correlation of wear and work in dual pivoted jaw crusher tests," Proceedings of the Institution of Mechanical Engineers, Part J: Journal of Engineering Tribology, vol. 234, no. 3, pp. 334-349, 2018.
[15] H. Murata, C. Masahiro, and H. Nagaharu, "Analysis of wear in impact crushers," The Resources Processing Society of Japan, vol. 38, no. 1, 1991.

[16] J. Zhang and F. Zhao, "Study on rotor load of vertical shaft impact crusher based on ADAMS," Journal of China Coal Society, vol. 34, no. 6, pp. 853-856, 2009.

[17] A. Zhu, S. Yang, Q. Li et al., "Simulation and measurement study of metro wheel wear based on the Archard model," Industrial Lubrication and Tribology, vol. 71, no. 2, pp. 284294, 2019.

[18] L. Ning and H. Fang, "Determination of hammer wear type of vertical shaft impact crusher," Journal of Huaqiao University, vol. 40, no. 5, pp. 305-311, 2019.

[19] G. Hu, Discrete Element Analysis and Simulation of Particle System, Wuhan University of Technology Press, Wuhan, China, 2010.

[20] P. He, H. Tang, and J. Wang, "Laser measurement system based on multi-joint and rapid casting detection of geomagic qualify," Laser Journal, vol. 35, no. 12, pp. 148-150, 2014.

[21] Z. Tao, C. Zhu, and M. He, "Test of $V$ shaped groove structure against rockfall based on orthogonal design," Journal of China Coal Society, vol. 42, no. 9, pp. 2307-2315, 2017.

[22] K. Gomathy, M. Balakrishnan, and R. Pandiselvam, "Nonlinear and multiple linear regression analysis of airflow resistance in multiplier onion," Journal of Food Process Engineering, vol. 42, no. 7, 2019.

[23] J. Li, Y. Xu, and X. Xie, "Influence of mining height on coal seam floor failure depth," Journal of China Coal Society, vol. 40, no. S2, pp. 303-310, 2015.

[24] Z. Sun and H. Lu, "Optimal traffic sensor layout model considering traffic big data," Journal of Tsinghua University (Science and Technology), vol. 56, no. 7, pp. 743-750, 2016. 